\title{
Rasgos de personalidad disfuncional en estudiantes universitarios de Buenos Aires
}

\author{
Juliana Beatriz Stover ${ }^{1}$
}

\begin{abstract}
RESUMEN
En la sección III del DSM-5 se propone un modelo alternativo para evaluar los trastornos de personalidad, en cuyo criterio $B$ se postula un modelo dimensional de rasgos disfuncionales de personalidad compuesto por cinco dominios y 25 rasgos. El objetivo de este trabajo fue analizar dichos dominios y rasgos en estudiantes universitarios de Buenos Aires. La muestra se compuso de 453 estudiantes universitarios de instituciones públicas y privadas $(52.30 \%$ mujeres, $47.70 \%$ varones) con edades comprendidas entre los 18 y 35 años $(M=24.26, D E=3.73)$. Se recolectaron datos con una encuesta sociodemográfica y de datos académicos básicos, así como con el Inventario para Trastornos de la Personalidad para el DSM-5 (PID-5). Para analizar diferencias en los dominios y rasgos según sexo y tipo de carrera se llevaron a cabo análisis de MANOVA. Se calcularon correlaciones $r$ de Pearson para examinar la asociación de los dominios y rasgos con el rendimiento académico. Se halló que las mujeres presentaron medias superiores Afecto Negativo, Ansiedad y Labilidad Emocional, y los hombres, en Antagonismo, Búsqueda de Atención, Insensibilidad, Engaño, Grandiosidad, Manipulación, Toma de Riesgo y Excentricidad. Examinado diferencias según tipo de carrera, se detectó un patrón de medias inferiores en los alumnos de Ciencias Humanas. Al analizar el vínculo con el rendimiento académico, se encontraron asociaciones negativas con el índice de aprobación, mientras que positivas con los índices de aplazos, asignaturas libres y recursadas. En conclusión, se efectuó una primera aproximación al estudio del modelo de rasgos disfuncionales en población local universitaria, analizado su vínculo con variables sociodemográficas y académicas.
\end{abstract}

Palabras Clave: trastornos de personalidad, DSM-5, PID-5, estudiantes universitarios, rendimiento académico.

\section{Dysfunctional personality traits in university students of Buenos Aires}

\begin{abstract}
Section III of the DSM-5 proposes an alternative model to assess personality disorders, in which criterion B postulated a dimensional model of dysfunctional personality traits composed of five domains and 25 traits. The aim of this paper was to analyze these domains and traits in university students of Buenos Aires. The sample consisted of 453 university students from public and private institutions (52.30\% female, $47.70 \%$ male) aged between 18 and 35 years $(M=24.26, S D=3.73)$. Data were collected using a survey of sociodemographic and basic academic data; as well as the Personality Inventory for DSM-5 (PID-5). In order to analyze differences in domains and traits according to sex and type of career, MANOVA analyzes were carried out. Pearson's $r$ correlations were calculated to examine the association of domains and traits with academic performance. It was found that women presented higher averages in Negative Affectivity, Anxiety and Lability Emotion and men in Antagonism, Attention Search, Insensitivity, Deception, Grandiosity, Manipulativeness, Risk Taking, and Eccentricity. Examined differences according to type of career a pattern of lower means was detected in the students of
\end{abstract}

${ }^{1}$ Consejo Nacional de Investigaciones Científicas y Técnicas (CONICET), Universidad de Buenos Aires, Argentina; stoverjuliana@gmail.com; https://orcid.org/0000-0003-2120-1031 
Human Sciences. When analyzing the relationship with academic performance, negative associations were found with the approval index, while positive with failed exams, failed subjects and recurring subjects. In conclusion, a first approach to the study of the model of dysfunctional traits in the local university student population was made, analyzing its link with sociodemographic and academic variables.

Keywords: personality disorders, DSM-5, PID-5, university students, academic performance.

En la quinta versión del Manual Diagnóstico y Estadístico de los Trastornos Mentales (DSM-5; American Psychiatric Association, 2013a) coexisten dos clasificaciones de los trastornos de personalidad (TP). En la Sección II se mantienen los criterios diagnósticos de la edición anterior y en la Sección III se propone un modelo híbrido categorial-dimensional, el cual en las investigaciones sobre personalidad, emerge con uno de los temas de mayor debate (Krueger, 2013). En el criterio B de dicho abordaje alternativo se plantean rasgos disfuncionales de personalidad. La principal hipótesis propuesta en este enfoque es la continuidad entre los rasgos de personalidad disfuncional y los rasgos de personalidad normal del modelo de los Cinco Factores (Costa \& McCrae, 1985), que describe la personalidad en términos de la combinación de los rasgos de neuroticismo, extraversión, apertura a la experiencia, afabilidad y responsabilidad (Gore \& Widiger, 2013; Widiger, 2011). De este modo, en el modelo se postulan cinco grandes dominios de personalidad disfuncional denominados afecto negativo, desapego, antagonismo, desinhibición y psicoticismo, los cuales, a su vez, se componen de 25 rasgos de personalidad disfuncional.

El afecto negativo se caracteriza por experiencias frecuentes e intensas de emociones negativas, así como por sus correspondientes manifestaciones comportamentales. Incluye los rasgos específicos de inestabilidad, ansiedad, inseguridad de separación, sumisión, perseveración, depresión, desconfianza, afectividad restringida y hostilidad. Se corresponde con el neuroticismo. El desapego, polo disfuncional de la extraversión, implica la evitación de la experiencia socioemocional (ya sea de amigos o relaciones intimas); incluyendo la restricción de la experiencia y expresión de afectos. Integra rasgos tales como aislamiento, evitación de intimidad, anhedonia, depresión, recelo y afectividad restringida. El antagonismo constituye la vertiente disfuncional de la afabilidad; caracteriza a sujetos con un exagerado sentido de importancia, cuyos objetivos involucran obtener ventajas respecto de otras personas; exhibiendo falta de empatía con los demás. Agrupa los rasgos de manipulación, engaño, grandiosidad, búsqueda de atención, labilidad emocional y hostilidad. La desinhibición se define por conductas impulsivas orientadas a la satisfacción inmediata, sin considerar consecuencias futuras. Contiene rasgos tales como irresponsabilidad, impulsividad, distractibilidad, toma de riesgos y perfeccionismo. Constituye la versión desadaptativa de la responsabilidad. Por último, el psicoticismo describe comportamientos y cogniciones que resultan excéntricas o inusuales en relación con la cultura a la que el individuo pertenece. Engloba los rasgos de creencias y experiencias inusuales, excentricidad y desregulación perceptual. Se postula como opuesto a la apertura a la experiencia (American Psychiatric Association, 2013a). En relación con este último par (psicoticismo-apertura a la experiencia), si bien excede el objetivo de este trabajo, debe remarcarse que su vínculo así como 
operacionalización tradicional de apertura a la experiencia se encuentran cuestionados (e.g., DeYoung, Grazioplene, \& Peterson, 2012; Góngora \& Castro Solano, 2017).

Los trabajos iniciales en torno a este modelo se focalizaron en la adaptación de instrumentos de evaluación, tales como el Inventario para Trastornos de la Personalidad para el DSM-5 -Personality Inventory for DSM-5; PID-5- (Al-Dajani, Gralnick \& Bagby, 2015; American Psychiatric Association, 2013b; Krueger et al., 2012), y el análisis del continuo de rasgos de personalidad disfuncional y normal (e.g., De Fruyt et al., 2013; Thomas et al., 2013).

Con relación al análisis de los rasgos disfuncionales de personalidad y su relación a otras variables, así como en ámbitos aplicados, se detecta menor cantidad de trabajos. Caracterizando los rasgos según variables sociodemográficas, Bastiaens et al. (2015) hallaron que las mujeres presentan valores significativamente superiores a los de los varones en afecto negativo y desinhibición, mientras que los hombres exhibían mayor antagonismo. A su vez, reportaron una relación negativa entre edad y los dominios de antagonismo, desinhibición y psicoticismo, así como una asociación inversa entre los años de educación y desinhibición. Considerando que las propiedades psicométricas del instrumento fueron estudiadas en población clínica y general (e.g., Al-Dajani et al., 2015), también se examinó la utilidad diagnóstica del modelo, reportándose que los puntajes del PID-5 diferencian a la población clínica de la general, encontrándose puntajes significativamente más elevados en el primer grupo (Bach, Maples-Keller, Bo \& Simonsen, 2016; Gutiérrez et al., 2015; Heath, Drvaric, Hendershot, Quilty \& Bagby, 2018). Sin embargo, a excepción de los estudios psicométricos, el análisis de los rasgos disfuncionales en otros ámbitos de aplicación así como en relación con otras variables -tales como las sociodemográficas o académicas- constituye un área de vacancia. Por ello, este trabajo tiene como objetivo general analizar los rasgos disfuncionales de personalidad en el ámbito educativo universitario, y analizar diferencias según sexo, tipo de carrera así como su vínculo con el desempeño académico.

En las investigaciones sobre variables asociadas al ajuste a la vida académica, ha sido ampliamente afirmado que las variables no intelectivas tienen importancia para explicar el desempeño académico (e.g., Richardson, Abraham \& Bond, 2012). Hasta la fecha, en la revisión realizada de la literatura, no se encontraron trabajos científicos que estudien la vinculación entre los desórdenes de personalidad propuestos por el modelo de personalidad evaluados con el PID-5 y el rendimiento académico. Por ello, deben reseñarse estudios que examinen constructos vinculados, tales como: a) la presencia de características de los trastornos de personalidad de la sección II del DSM-5 (American Psychiatric Association, 2013a), la cual es coincidente con la edición anterior; b) la sintomatología psicopatológica en general aunque se consideran estados sintomáticos y no rasgos de personalidad, fueron incluidos considerando una arista secundaria para analizar la salud mental de los estudiantes-; y c) el vínculo con el modelo de los Cinco Factores (Costa \& McCrae, 1985) -que, como ya se mencionó, constituye su opuesto teórico en términos de personalidad normal-.

Comenzando por la presencia de sintomatología asociada a TP, en distintas muestras de estudiantes universitarios se encontró que el $21.5 \%$ de los alumnos presentó características de algún TP (Micin \& Bagladi, 2011), 
hallándose que los mismos reportaban mayor sintomatología en comparación con la población general (Chabrol, Rousseau, Callahan \& Hyler, 2007; Ekselius, Tillfors, Furmark, \& Frederikson, 2001). Con relación a las diferencias según sexo, en términos generales se detectó que los hombres exhiben mayor cantidad de trastornos y, al considerar cada diagnóstico por separado, los varones presentan características de trastornos esquizoide, narcisista y obsesivo-compulsivo, mientras que las mujeres demuestran con mayor frecuencia los trastornos limítrofe e histriónico (Micin \& Bagladi, 2011). Al evaluar las características del recorrido por el nivel universitario, Krajniak, Pievsky, Eisen y McGrath (2017) detectaron bajo ajuste a la vida académica en los estudiantes con rasgos de trastornos paranoide, esquizoide, esquizotípico, antisocial, limítrofe, evitativo y dependiente. En la misma línea, Hunt, Eisenberg y Kilbourne (2010) informaron que alumnos con trastorno antisocial tenian menores posibilidades de graduarse, mientras que Bagge et al. (2004) hallaron que las características de trastorno limítrofe se asociaban con menor rendimiento académico.

Al analizar la sintomatologia en estudiantes universitarios, al igual que lo mencionado para las características de los TP, se informaron valores elevados en diversos indicadores, como, por ejemplo, la presencia de signos de depresión y ansiedad entre el 15\% y el 25\% de los estudiantes (e.g., Arrivillaga Quintero, Cortés Garcia, Goicochea Jiménez \& Lozano Ortíz, 2004; Eisenberg, Gollust, Golberstein \& Hefner, 2007). Y, al examinar las diferencias según sexo, se hallaron puntajes superiores de ansiedad, depresión y somatizaciones en mujeres, mientras que ideación paranoide más alta en varones (Agudelo Vélez, Casadiegos Garzón \& Sánchez Ortíz, 2008; Casullo, Morandi \& Donati, 2006). Otros trabajos tuvieron en cuenta las carreras que cursaban los educandos, hallándose rasgos autistas y esquizoides en estudiantes de ciencias comparados con los de humanidades (Choteau, Raynal, Goutaudier \& Chabrol, 2016). Con relación al ajuste a la vida académica, se encontraron resultados disímiles. En algunos estudios se reportó que mayor sintomatología psicopatológica se vinculaba a peor desempeño (Urquijo, Del Valle, Ané, Heredia \& Martínez, 2013), así como que los alumnos con bajo rendimiento académico presentaron indicadores de depresión mayor y riesgo suicida (Valencia-Molina, Pareja-Galvis \& Montoya Arenas, 2014). Sin embargo, otras investigaciones no detectaron vínculo entre la presencia de psicopatología y el desempeño (Campo Cabal \& Gutiérrez Segura, 2001; Stover \& Bruno, 2016).

Focalizados en los estudios en torno al modelo de los Cinco Factores (Costa \& McCrae, 1985), existen numerosas investigaciones debido a la vigencia y difusión de este marco conceptual. Al analizar diferencias según sexo, los hallazgos son similares, al indicar que las mujeres exhiben mayor neuroticismo y afabilidad que los hombres (Chapman, Duberstein, Sörensen \& Lyness, 2007; Kajoniusa \& Johnson, 2018; Rantanen, Metsäpelto, Feldt, Pulkkinen \& Kokko, 2007). Con relación al desempeño académico, mediante un meta-análisis Poropat (2009), encontró que el rendimiento se asociaba con afabilidad, apertura a la experiencia y responsabilidad, siendo esta última dimensión la que mayor importancia tuvo. En concordancia, Hakimi, Hejazi y Lavasani (2011) encontraron que las características de la personalidad explicaban el $48 \%$ de la varianza en el rendimiento académico y, de dicho porcentaje, la responsabilidad implicaba el 39\%. Smrtnik Vitulic y Prosen (2011) informaron que las correlaciones más elevadas entre las calificaciones y las dimensiones del modelo se presentaron con el factor de responsabilidad. 
Estudios recientes continúan aportando datos similares. Dumfart y Neubauer (2016) también hallaron que responsabilidad es el predictor del rendimiento no cognitivo más importante. Por su parte, Bergold y Steinmayr (2018) encontraron que puntajes altos en responsabilidad se vinculan con mejores calificaciones, mientras que valores bajos en neuroticismo se asocian al alto desempeño.

Por lo hasta aquí expuesto, al considerar la importancia de examinar factores no intelectivos vinculados al tránsito por la vida universitaria (e.g., Richardson et al., 2012) y que, entre ellos el modelo de los Cinco Factores ha demostrado su poder predictivo (Poropat, 2009), resulta de interés indagar si los rasgos disfuncionales de personalidad -postulados como opuestos a dicho modelo- guardan relación con variables académicas. Por ello este trabajo tiene los objetivos de: 1) examinar diferencias en los dominios y rasgos según sexo y carrera, y 2) analizar asociaciones entre los dominios y rasgos disfuncionales de personalidad y el rendimiento académico. Se hipotetiza que: 1) se detectarán diferencias según sexo y carrera en los dominios y rasgos; 2) los alumnos con peor desempeño presentarán puntajes superiores en los dominios y rasgos.

\section{MÉTODO}

\section{Participantes}

Mediante un muestreo intencional simple se recolectaron 477 casos, 7 de los cuales fueron descartados debido a que sus protocolos presentaban datos sociodemográficos incompletos, y respuestas al PID-5 con tendencias a responder en una sola dirección. Se eliminaron 17 casos por presentar casos atípicos uni- y multivariados en las 25 facetas y cinco dominios. Por lo tanto, la muestra final se compuso de 453 estudiantes universitarios con edades comprendidas entre los 18 y 35 años $(M=24.26 ; D E=3.73)$. Los porcentajes según sexo fueron similares (52.30\% mujeres, $47.70 \%$ varones). El $70.15 \%$ de los participantes trabajaba al momento de evaluación. El mayor porcentaje reportó percibirse en el nivel socio-económico medio $(73.58 \%)$, siguiéndole en porcentajes el medio-alto (14.12\%), medio-bajo (10.94\%). Los niveles bajo y alto agruparon a la misma cantidad de sujetos $(0.68 \%)$. En relación con sus datos académicos, el $84.30 \%$ cursaba sus estudios en una universidad de gestión pública y el $15.70 \%$, en una institución privada. Considerando la clasificación del Ministerio de Educación (2013), la distribución por tipo de carrera fue: $36.42 \%$ Ciencias Sociales, 30.68\% Ciencias Humanas, $22.07 \%$ Ciencias Aplicadas-Básicas y 10.83\% Ciencias de la Salud.

\section{Materiales}

- Encuesta de datos sociodemográficos y datos académicos básicos. Recoge información con preguntas cerradas sobre: sexo (femenino, masculino), edad en años, situación laboral (trabaja o no), nivel socioeconómico percibido (bajo, medio-bajo, medio, medio-alto, alto). También indaga universidad, facultad y carrera en curso, cantidad de asignaturas de la carrera en curso, así como cantidad de materias aprobadas, aplazadas, libres y recursadas. En el caso de la información académica, se trata de un formato estructurado en el cual los alumnos deben escribir el nombre de su universidad, facultad y carrera, y responder en formato numérico al resto de las preguntas. La 
encuesta de datos sociodemográfica fue diseñada para los estudios llevados a cabo en el marco de los proyectos en curso en el equipo de investigación.

- Inventario para Trastornos de la Personalidad para el DSM-5 Personality Inventory for DSM-5; PID-5- (American Psychiatric Association, 2013b; Krueger et al., 2012; Stover, Castro Solano \& Fernández Liporace, 2019). Consta de 220 items con respuesta likert de cuatro posiciones (Muy Falso o A menudo Falso = 0; A veces o Algo Falso $=1 ;$ A veces o Algo Verdadero $=2 ;$ Muy Cierto o A menudo Verdadero $=3$ ). Evalúa los ya mencionados 25 rasgos de personalidad disfuncionales y los cinco dominios. En la adaptación local con población general adulta se verificó su estructura pentafactorial mediante análisis factoriales exploratorios y se halló una consistencia interna adecuada para los dominios (alfas de Cronbach entre .85 y .93) y para 22 de las facetas (alfas de Cronbach entre .70 y .91).

\section{Procedimiento}

Se contactó a docentes de diversas universidades que participaron en investigaciones previas. Se recolectaron datos mediante administraciones colectivas en las aulas y horarios habituales de clases en siete universidades públicas y cuatro privadas. Los protocolos fueron completados en formato papel, con una duración promedio de 30 minutos. De acuerdo con lo determinado por el Consejo Nacional de Investigaciones Científicas y Técnicas en su Resolución D N 2857, mediante consentimiento informado se explicó a los participantes que se garantizarian la confidencialidad y el anonimato, informando sobre la posibilidad de abstenerse de responder o de interrumpir la evaluación en cualquier momento, así como que sus respuestas no tendrian influencia en su trayectoria académica.

\section{Análisis de datos}

Antes de responder a los objetivos, se detectaron los ítems sin respuesta, los cuales al no superar el 5\% fueron imputados por la media del item (Rial Boubeta, Varela Mallou \& Tejada, 2001). Posteriormente, se eliminaron 17 casos atípicos de acuerdo con los resultados de contrastes uni- y multivariados (diagramas de cajas y distancia de Mahalanobis).

Con relación a los análisis de datos, se calcularon análisis multivariados de la varianza (MANOVA) para examinar diferencias según sexo y tipo de carrera en los cinco dominios, por un lado, y en los 25 rasgos por otro. Se seleccionó este análisis para controlar el error de tipo I, considerando la asociación entre los dominios y rasgos reportada en estudios previos (Stover, Castro Solano \& Fernández Liporace, 2019) así como la relación teórica entre los mismos (American Psychiatric Association, 2013a). En relación con los supuestos requeridos para el análisis, el número de observaciones en cada celda fue superior al número de variables dependientes, superando a su vez el criterio mínimo de 20 casos por celda establecido por Hair, Anderson, Tatham y Black (1999). Además, las medidas dependientes se hallaron correlacionadas con la matriz de correlación, detectándose resultados estadísticamente significativos en prueba de esfericidad de Bartlett tanto para los dominios $\left(X^{2}=562.10 ; 10 g l ; p=.000\right)$ como para los rasgos $\left(X^{2}=6002.34\right.$, $300 \mathrm{gl} ; p<.001)$. Si bien no se cumplió con el supuesto de normalidad, la violación de este no influye en los análisis de tamaños muestrales grandes como es el caso de este trabajo- (Hair et al., 1999). Como complemento se calcularon los valores de asimetría y curtosis, los cuales fueron inferiores al 
punto de corte de \pm 2 . Considerando que la homocedasticidad estimada mediante la prueba de M-Box y el contraste de Levene en algunos análisis resultaba estadísticamente significativa, se escogió la Traza de Hotelling por resultar más robusta ante el incumplimiento de algunos supuestos (Finch \& French, 2013). Para el análisis post-hoc, se utilizó el contraste Tuckey debido a su potencia (Pardo \& San Martín, 1999).

Se calcularon correlaciones $r$ de Pearson para indagar la asociación entre el rendimiento académico, los dominios y rasgos. Para la interpretación de los tamaños del efecto se consideraron para los puntos de corte establecidos por Cohen (1988) tanto para la interpretación del $\eta^{2}$ (pequeño $=.01$; moderado $=$ .05 ; grande $=.13$ ) como para la del coeficiente $r$ de Pearson (pequeño $=.10$; moderado $=.24$; grande $=.37$ ) .

Al momento de medir el rendimiento académico se dispone de múltiples operacionalizaciones (Garbanzo Vargas, 2014; Richardson et al., 2012). Para este trabajo se consideró su acepción amplia que involucra el éxito, retraso y abandono de los estudios, tomando en cuenta no solo la aprobación (es decir la eficiencia del proceso educativo), sino la eficacia en función del tiempo que ello implica (Rodríguez, Fita \& Torrado, 2004). Por lo tanto, se calcularon cuatro índices. El primero consistió en la división de la cantidad de asignaturas aprobadas sobre el total de materias que tiene la carrera, lo cual brinda información sobre el avance en la carrera, es decir, el adecuado rendimiento académico. Los restantes índices se compusieron por la división de las asignaturas aplazadas, libres y recursadas, sobre el total de materias aprobadas en los tres casos, es decir, el fracaso y retraso en los estudios.

Todos los análisis se realizaron con el software PASW Statistics, versión 18.0 (SPSS Inc., 2009).

\section{RESULTADOS}

Se analizaron los estadísticos descriptivos para los dominios y rasgos, detectándose valores inferiores a \pm 2 en asimetría y curtosis (tabla 1).

Con el objetivo de examinar diferencias en los cinco dominios se calculó un MANOVA con un diseño 2 (Sexo $=$ Mujeres y Varones) $\mathrm{x} 4$ (Carrera $=$ Aplicadas-Básicas, Ciencias de la Salud, Ciencias Humanas, Ciencias Sociales) $\times 5$ (Dominios $=$ Afecto Negativo, Desapego, Antagonismo, Desinhibición, Psicoticismo). No se observó interacción entre el Sexo y el tipo de Carrera $\left(T^{2}=0.04, F(15,1321)=1.44, p=.120, \eta^{2}=.01 ; M-B o x=170.46, p\right.$ $<.001$ ), por lo tanto se calcularon diferencias en los dominios según sexo por un lado y según tipo de carrera por el otro. En relación con los primeros, se hallaron diferencias con tamaño del efecto grande $\left(T^{2}=0.17, F(5,447)=15.39\right.$, $\left.p<.001, \eta^{2}=.14 ; M-B o x=26.95, p=.032\right)$, en donde las mujeres presentaron medias superiores en Afecto Negativo y los hombres en Antagonismo. El tamaño del efecto fue bajo en el primer caso y moderado en el segundo (tabla 2). 
Tabla 1.

Dominios y rasgos. Estadísticos descriptivos.

\begin{tabular}{lcccc}
\hline & $\boldsymbol{M}$ & $\boldsymbol{D E}$ & Asimetría & Curtosis \\
\hline Afecto Negativo & 24.13 & 11.33 & 0.45 & -0.33 \\
Desapego & 15.34 & 9.71 & 0.80 & 0.10 \\
Antagonismo & 12.64 & 8.68 & 0.74 & 0.03 \\
Desinhibición & 19.00 & 9.88 & 0.40 & -0.15 \\
Psicoticismo & 17.00 & 14.35 & 1.11 & 0.85 \\
Anhedonia & 5.73 & 3.95 & 0.94 & 0.39 \\
Ansiedad & 11.53 & 5.64 & 0.38 & -0.57 \\
Búsqueda de atención & 7.76 & 5.10 & 0.56 & -0.23 \\
Insensibilidad & 5.89 & 5.46 & 1.25 & 1.43 \\
Engaño & 5.89 & 4.12 & 0.94 & 0.65 \\
Depresión & 5.17 & 5.47 & 1.30 & 1.10 \\
Distractibilidad & 8.48 & 5.39 & 0.34 & -.051 \\
Excentricidad & 8.55 & 8.03 & 1.13 & 0.70 \\
Labilidad emocional & 8.08 & 4.48 & 0.40 & -0.47 \\
Grandiosidad & 3.10 & 3.03 & 1.07 & 0.95 \\
Hostilidad & 11.16 & 5.35 & 0.31 & -0.55 \\
Impulsividad & 6.01 & 4.12 & 0.49 & -0.37 \\
Evitación de la intimidad & 3.76 & 2.91 & 1.09 & 1.35 \\
Irresponsabilidad & 4.51 & 3.14 & 0.86 & 0.68 \\
Manipulación & 3.64 & 2.94 & 0.59 & -0.49 \\
Desregulación perceptual & 5.30 & 4.74 & 1.25 & 1.45 \\
Perseveración & 8.45 & 5.01 & 0.35 & -0.37 \\
Afectividad restringida & 6.97 & 3.87 & 0.44 & -0.04 \\
Perfeccionismo & 10.88 & 6.62 & 0.42 & -.042 \\
Toma de riesgos & 17.23 & 6.37 & 0.31 & -0.17 \\
Inseguridad de separación & 4.52 & 3.83 & 0.98 & 0.54 \\
Sumisión & 3.49 & 2.36 & 0.61 & 0.13 \\
Recelo & 7.11 & 3.55 & 0.50 & -0.10 \\
Creencias y Experiencias Inusuales & 3.15 & 3.63 & 1.31 & 1.26 \\
Aislamiento & 5.85 & 5.24 & 0.93 & 0.14 \\
\hline & & & &
\end{tabular}

Tabla 2.

Dominios del PID- 5 según sexo. MANOVA.

\begin{tabular}{lccccc}
\hline & \multicolumn{2}{c}{$M(D E)$} & Levene & \multirow{2}{*}{} \\
\cline { 2 - 4 } & Mujeres & Varones & $p$ & & $\eta^{2}$ \\
\hline Afecto Negativo & $26.33(11.61)$ & $21.70(10.51)$ & .09 & $19.64^{* *}$ & .04 \\
Desapego & $14.51(9.81)$ & $16.24(9.54)$ & .76 & 3.58 & 0 \\
Antagonismo & $10.62(7.37)$ & $14.86(9.46)$ & .00 & $28.5^{* *}$ & .06 \\
Desinhibición & $19.19(10.16)$ & $18.79(9.58)$ & .37 & 0.17 & .00 \\
Psicoticismo & $16.02(13.88)$ & $18.08(14.81)$ & .21 & 2.34 & 0 \\
\hline
\end{tabular}

${ }^{* *} p<.01 ;{ }^{*} p<.05$

Se encontraron diferencias según tipo de carrera con tamaño del efecto bajo $\left(T^{2}=0.09, F(15,1331)=2.68, p<.001, \eta^{2}=.02 ; M-B o x=61.46, p=.068\right)$ exhibiendo los alumnos de Ciencias Aplicadas-Básicas mayores medias que los estudiantes de Ciencias Humanas en Afecto Negativo, Desinhibición y Desapego. El tamaño del efecto fue bajo en todos los casos -tabla 3-. 
Tabla 3.

Dominios del PID- 5 según tipo de carrera. MANOVA.

\begin{tabular}{|c|c|c|c|c|c|c|c|}
\hline & \multicolumn{4}{|c|}{$M(D E)$} & \multirow{2}{*}{$\begin{array}{l}\text { Levene } \\
\qquad p\end{array}$} & \multirow[b]{2}{*}{$F$} & \multirow[b]{2}{*}{$\eta^{2}$} \\
\hline & $\begin{array}{l}\text { Aplicadas- } \\
\text { Básicas }\end{array}$ & $\begin{array}{c}\text { Ciencias de la } \\
\text { Salud }\end{array}$ & $\begin{array}{l}\text { Ciencias } \\
\text { Humanas }\end{array}$ & $\begin{array}{l}\text { Ciencias } \\
\text { Sociales }\end{array}$ & & & \\
\hline Afecto Negativo & 24.11(10.13) & $25.69(12.40)$ & 24.99(11.08) & $22.95(11.85)$ & .26 & 1.17 & 0 \\
\hline Desapego & $17.74(9.98)^{\AA}$ & $17(10.12)^{A B}$ & $13.83(8.63)^{B}$ & $14.66(10.02)^{A B}$ & .12 & $3.98^{* *}$ & .02 \\
\hline Antagonismo & $13.80(8.72)$ & $12.96(8.75)$ & $10.76(7.66)$ & 13.42(9.25) & .13 & 3.27 & .02 \\
\hline Desinhibición & $20.71(9.72)^{A}$ & $20.27(9.74)^{A ́ B}$ & $16.71(9.04)^{B}$ & $19.51(10.43)^{A B}$ & .53 & $3.96^{* *}$ & .02 \\
\hline Psicoticismo & $20.34(14.56)^{\mathrm{A}}$ & $18.47(16.16)^{\mathrm{AB}}$ & $15.43(14.73)^{\mathrm{B}}$ & $15.87(13.04)^{A B}$ & .30 & $2.90^{*}$ & .01 \\
\hline
\end{tabular}

Al examinar diferencias en los rasgos disfuncionales de personalidad según Sexo y Carrera, se efectuó un MANOVA con un diseño 2 (Sexo = Mujeres y Varones) $\times 4$ (Carrera = Aplicadas-Básicas, Ciencias de la Salud, Ciencias Humanas, Ciencias Sociales). No se observó interacción entre el Sexo y el tipo de Carrera $\left(T^{2}=0.20, F(75,1259)=1.12, p=.229, \eta^{2}=.01 ; M-B o x=\right.$ $2930.80 ; p<.001)$. Por lo tanto, se analizaron los factores fijos por separado. Se calculó un MANOVA (Rasgos $=25 \times$ Sexo $=2$ ), verificándose diferencias estadísticamente significativas entre los vectores de medias con tamaño del efecto grande $\left(T^{2}=0.32, F(25,427)=5.61, p<.001, \eta^{2}=.24 ; M-B o x=407.32, p\right.$ $=.013)$. Las diferencias fueron a favor de las mujeres en Ansiedad, Labilidad Emocional e Impulsividad, mientras que los hombres reportaron medias superiores en Búsqueda de Atención, Insensibilidad, Engaño, Excentricidad, Grandiosidad, Manipulación, y Afectividad Restringida y Toma de Riesgo. Los tamaños del efecto fueron moderados en Engaño y Labilidad Emocional, y bajos en los restantes -tabla 4-.

Tabla 4.

Rasgos del PID- 5 según sexo. MANOVA.

\begin{tabular}{|c|c|c|c|c|c|}
\hline & \multicolumn{2}{|c|}{$M(D E)$} & \multirow{2}{*}{$\begin{array}{c}\text { Levene } \\
\qquad p\end{array}$} & \multirow{2}{*}{$F$} & \multirow{2}{*}{$\eta^{2}$} \\
\hline & Mujeres & Varones & & & \\
\hline Anhedonia & $5.48(3.97)$ & 6(3.92) & .81 & 1.91 & .004 \\
\hline Ansiedad & $12.41(5.89)$ & $10.56(5.19)$ & .05 & $12.54^{\star *}$ & .027 \\
\hline Búsqueda de atención & $7.04(5.16)$ & $8.55(4.92)$ & .52 & $10.05^{\star *}$ & .022 \\
\hline Insensibilidad & $4.89(4.68)$ & $6.99(6.03)$ & .00 & $17.29^{* *}$ & .037 \\
\hline Engaño & $4.89(3.46)$ & $6.99(4.50)$ & .00 & $31.13^{* *}$ & .065 \\
\hline Depresión & $5.19(5.42)$ & $5.16(5.53)$ & .56 & 0.00 & .000 \\
\hline Distractibilidad & $8.40(5.56)$ & $8.56(5.20)$ & .38 & 0.09 & .000 \\
\hline Excentricidad & $7.47(7.57)$ & $9.75(8.36)$ & .01 & $9.24^{* *}$ & .020 \\
\hline Labilidad emocional & $9.16(4.60)$ & $6.90(4.05)$ & .02 & $30.33^{* *}$ & .063 \\
\hline Grandiosidad & $2.64(2.64)$ & $3.61(3.34)$ & .00 & $11.84^{* *}$ & .026 \\
\hline Hostilidad & $11.46(5.28)$ & $10.84(5.42)$ & .44 & 1.50 & .003 \\
\hline Impulsividad & $6.55(4.22)$ & $5.42(3.94)$ & .53 & $8.57^{* *}$ & .019 \\
\hline Evitación de la intimidad & $3.54(2.85)$ & $4(2.96)$ & .41 & 2.88 & .006 \\
\hline Irresponsabilidad & $4.24(2.99)$ & $4.81(3.26)$ & .11 & 3.79 & .008 \\
\hline Manipulación & $3.08(2.71)$ & $4.26(3.07)$ & .00 & $18.64^{* *}$ & .040 \\
\hline Desregulación perceptual & $5.37(4.49)$ & $5.22(5.01)$ & .17 & 0.11 & .000 \\
\hline Perseveración & $8.56(4.89)$ & $8.34(5.14)$ & .30 & 0.21 & .000 \\
\hline Afectividad restringida & $6.33(3.97)$ & $7.67(3.65)$ & .08 & $13.73^{* *}$ & .030 \\
\hline Perfeccionismo & $11(6.70)$ & $10.76(6.55)$ & .46 & 0.14 & .000 \\
\hline Toma de riesgo & $16.29(6.04)$ & $18.26(6.58)$ & .12 & $11.05^{\star *}$ & .024 \\
\hline Inseguridad & $4.76(3.75)$ & $4.25(3.91)$ & .90 & 2.06 & .005 \\
\hline Sumisión & $3.51(2.42)$ & $3.46(2.31)$ & .39 & 0.05 & .000 \\
\hline Recelo & $7(3.62)$ & $7.23(3.48)$ & .61 & 0.46 & .001 \\
\hline Creencias y experiencias inusuales & $3.18(3.66)$ & $3.12(3.61)$ & .59 & 0.03 & .000 \\
\hline Aislamiento & $5.49(5.45)$ & $6.24(5)$ & .25 & 2.29 & .005 \\
\hline
\end{tabular}

${ }^{* *} p<.01 ;{ }^{*} p<.05$. 
Al calcular diferencias en los rasgos disfuncionales según tipo de carrera se realizó un MANOVA (Rasgos $=25 \times$ Carrera $=4$ ), en el que se hallaron diferencias estadísticamente significativas entre los vectores de media con tamaño del efecto moderado $\left(T^{2}=0.29, F(75,1271)=1.68, p<.001, \eta^{2}=.09\right.$; $M-B o x=1365.26, p<.001)$. Los alumnos de Ciencias Humanas reportaron medias menores en Insensibilidad y Distractibilidad (respecto de los de Ciencias Aplicadas-Básicas), Grandiosidad, Hostilidad y Recelo (comparados con los estudiantes de Ciencias de la Salud), Afectividad Restringida y Aislamiento (respecto de los estudiantes de Ciencias Aplicadas-Básicas y Ciencias de la Salud) y Toma de Riesgos (comparados con todos los grupos). El tamaño del efecto fue bajo en todos los casos. Los resultados se detallan en la tabla 5 .

Tabla 5

Rasgos del PID- 5 según tipo de carrera. MANOVA

\begin{tabular}{|c|c|c|c|c|c|c|c|}
\hline & \multicolumn{4}{|c|}{$M(D E)$} & \multirow{2}{*}{$\begin{array}{l}\text { Levene } \\
\quad p\end{array}$} & \multirow[b]{2}{*}{$F$} & \multirow[b]{2}{*}{$\eta^{2}$} \\
\hline & $\begin{array}{l}\text { Aplicadas- } \\
\text { Básicas }\end{array}$ & $\begin{array}{l}\text { Ciencias de } \\
\text { la Salud }\end{array}$ & $\begin{array}{l}\text { Ciencias } \\
\text { Humanas }\end{array}$ & $\begin{array}{l}\text { Ciencias } \\
\text { Sociales }\end{array}$ & & & \\
\hline \multirow{3}{*}{$\begin{array}{l}\text { Anhedonia } \\
\text { Ansiedad } \\
\text { Búsqueda de } \\
\text { atención }\end{array}$} & $6.27(3.80)$ & $5.98(4.37)$ & $5.45(3.62)$ & $5.55(4.18)$ & .21 & 1.02 & 0 \\
\hline & $11.83(5.24)$ & $12.18(6.16)$ & $11.89(5.54)$ & 10.84(5.79) & .52 & 1.32 & 0 \\
\hline & $8.03(4.80)$ & $7.29(5.46)$ & $7.61(5.34)$ & $7.85(4.99)$ & .56 & .29 & 0 \\
\hline Insensibilidad & $6.91(5.32)^{\mathrm{A}}$ & $6.41(5.66)^{\mathrm{AB}}$ & $4.59(4.69)^{\mathrm{B}}$ & $6.22(5.91)^{A B}$ & .03 & $4.21^{* *}$ & .02 \\
\hline Engaño & $6.53(3.96)$ & $5.73(3.85)$ & $5.05(3.60)$ & $6.25(4.59)$ & .03 & 3.22 & .02 \\
\hline Depresión & $5.45(5.87)$ & $5.59(6.33)$ & $5.32(5.44)$ & $4.75(4.96)$ & .27 & 0.54 & 0 \\
\hline Distractibilidad & $9.89(5.37)^{\mathrm{A}}$ & $9.06(5.52)^{\mathrm{AB}}$ & $7.56(4.93)^{B}$ & $8.22(5.58)^{A B}$ & .34 & $4.02^{* *}$ & .02 \\
\hline Excentricidad & $10.56(8.37)$ & $9.18(9.07)$ & 7.82(7.91) & $7.77(7.42)$ & .36 & 3.13 & .02 \\
\hline $\begin{array}{l}\text { Labilidad } \\
\text { emocional }\end{array}$ & $7.68(4.29)$ & $8.78(4.49)$ & $8.58(4.66)$ & $7.70(4.42)$ & .91 & 1.61 & .01 \\
\hline Grandiosidad & $3.41(3.44)^{\mathrm{AB}}$ & $3.57(3.39)^{\mathrm{B}}$ & $2.39(2.48)^{\mathrm{A}}$ & $3.38(2.99)^{\mathrm{AB}}$ & .03 & $3.84^{*}$ & .02 \\
\hline Hostilidad & $11.51(5.38)^{\mathrm{AB}}$ & $12.98(6.19)^{\mathrm{B}}$ & $10.07(4.71)^{\mathrm{A}}$ & $11.33(5.44)^{\mathrm{AB}}$ & .03 & $4.07^{* *}$ & .02 \\
\hline Impulsividad & 5.98(4.18) & $6.43(4.18)$ & 5.19(3.77) & $6.60(4.27)$ & .44 & 3.18 & .02 \\
\hline $\begin{array}{l}\text { Evitación de la } \\
\text { intimidad }\end{array}$ & $4.28(3.29)$ & $3.80(2.76)$ & $3.78(2.92)$ & $3.42(2.66)$ & .14 & 1.83 & .01 \\
\hline Irresponsabilidad & $4.84(3.09)$ & $4.78(3.28)$ & $3.96(2.73)$ & $4.69(3.39)$ & .06 & 2.08 & .01 \\
\hline Manipulación & $3.86(2.94)$ & $3.65(2.94)$ & $3.32(2.81)$ & $3.78(3.06)$ & .48 & 0.84 & 0 \\
\hline $\begin{array}{l}\text { Desregulación } \\
\text { perceptual }\end{array}$ & $6.08(4.78)$ & $5.59(5.16)$ & $4.96(5.02)$ & $5.01(4.32)$ & .26 & 1.39 & 0 \\
\hline \multirow{2}{*}{$\begin{array}{l}\text { Perseveración } \\
\text { Afectividad } \\
\text { restringida }\end{array}$} & $9.46(5.19)$ & $8.88(5.32)$ & $7.81(4.82)$ & $8.26(4.89)$ & .64 & 2.33 & .01 \\
\hline & $7.99(4.19)^{\mathrm{A}}$ & $7.88(4.15)^{\mathrm{A}}$ & $5.89(3.30)^{\mathrm{B}}$ & $6.99(3.83)^{\mathrm{AB}}$ & .11 & $7.05^{* *}$ & .04 \\
\hline \multirow{3}{*}{$\begin{array}{l}\text { Perfeccionismo } \\
\text { Toma de riesgo } \\
\text { Inseguridad }\end{array}$} & $11.70(6.55)$ & $11.86(7.14)$ & $10.14(6.35)$ & $10.72(6.70)$ & .79 & 1.47 & .01 \\
\hline & $17.77(6.31)^{\mathrm{A}}$ & $18.73(6.04)^{A}$ & $15.41(5.75)^{B}$ & $17.99(6.72)^{\mathrm{A}}$ & .33 & $5.89^{* *}$ & .03 \\
\hline & $4.60(3.94)$ & $4.73(3.81)$ & $4.52(3.58)$ & $4.40(4.01)$ & .52 & 0.11 & 0 \\
\hline \multirow{2}{*}{$\begin{array}{l}\text { Sumisión } \\
\text { Recelo }\end{array}$} & $3.64(2.49)$ & $3.73(2.82)$ & $3.76(2.26)$ & $3.08(2.19)$ & .08 & 2.55 & .01 \\
\hline & $7.28(3.48)^{\mathrm{AB}}$ & $8.24(3.91)^{A}$ & $6.47(3.48)^{B}$ & $7.20(3.47)^{\mathrm{AB}}$ & .72 & $3.30^{*}$ & .02 \\
\hline $\begin{array}{l}\text { experiencias } \\
\text { inusuales }\end{array}$ & $3.70(3.71)$ & $3.69(3.94)$ & $2.65(3.47)$ & $3.08(3.59)$ & .31 & 2.04 & .01 \\
\hline Aislamiento & $7.19(5.48)^{\mathrm{A}}$ & $7.22(5.48)^{\mathrm{A}}$ & $4.59(4.26)^{\mathrm{B}}$ & $5.69(5.53)^{\mathrm{AB}}$ & .00 & $6.22^{* *}$ & .04 \\
\hline
\end{tabular}

Letras diferentes indican grupos diferentes en el análisis post-hoc Tuckey; ${ }^{* *} p<.01 ;{ }^{*} p<.05$

Al analizar las correlaciones entre los dominios, rasgos e índices -tabla 6-, se hallaron diversas correlaciones estadísticamente significativas bajas, las cuales resultaron negativas entre el índice de aprobación, mientras que en su mayoría positivas con los demás índices -tabla 6-. En el índice de aprobación 
los coeficientes más altos se detectaron con Hostilidad $(r=-.21)$ e Impulsividad $(r=-.21)$. En relación con el índice de aplazos, los valores más elevados se hallaron con Distractibilidad $(r=.16)$; en el índice de asignaturas libres con Hostilidad $(r=.14)$ e Impulsividad $(r=.14)$. El indice de asignaturas recursadas el coeficiente más elevado fue en su vínculo con Distractibilidad ( $r$ $=.21)$. Desinhibición y Distractibilidad fueron el dominio y el rasgo que presentaron mayor número de correlaciones significativas.

Tabla 6.

Rendimiento académico, dominios y rasgos del PID- 5. Asociaciones.

\begin{tabular}{|c|c|c|c|c|}
\hline & $\begin{array}{c}\text { Aprobación/ } \\
\text { Total }\end{array}$ & $\begin{array}{c}\text { Aplazol } \\
\text { Aprobación }\end{array}$ & $\begin{array}{c}\text { Libre/ } \\
\text { Aprobación }\end{array}$ & $\begin{array}{c}\text { Recurso/ } \\
\text { Aprobación }\end{array}$ \\
\hline Afecto Negativo & $-.10^{*}$ & -.01 & .03 & .03 \\
\hline Desapego & $-.11^{*}$ & .09 & .04 & $.16^{*}$ \\
\hline Antagonismo & -.04 & .05 & .02 & .07 \\
\hline Desinhibición & $-.17^{* *}$ & $.13^{* *}$ & .07 & $.15^{\star *}$ \\
\hline Psicoticismo & $-.14^{\star *}$ & .03 & .07 & .09 \\
\hline Anhedonia & -.05 & .09 & -.01 & .12 \\
\hline Ansiedad & $-.10^{*}$ & .01 & .04 & .03 \\
\hline Búsqueda de atención & -.07 & .00 & .01 & -.02 \\
\hline Insensibilidad & -.07 & $.11^{\star *}$ & $.11^{*}$ & .08 \\
\hline Engaño & -.03 & .05 & -.03 & .05 \\
\hline Depresión & $-.10^{*}$ & .06 & -.04 & $.15^{*}$ \\
\hline Distractibilidad & $-.10^{*}$ & $.16^{* *}$ & .00 & $.21^{*}$ \\
\hline Excentricidad & $-.10^{*}$ & .04 & .06 & .11 \\
\hline Labilidad emocional & -.08 & -.03 & .00 & .05 \\
\hline Grandiosidad & -.02 & .08 & .02 & .13 \\
\hline Hostilidad & $-.21^{\star *}$ & .08 & $.14^{\star *}$ & .10 \\
\hline Impulsividad & $-.21^{* *}$ & .03 & $.14^{* *}$ & .03 \\
\hline Evitación de la intimidad & $-.10^{*}$ & .01 & .04 & .11 \\
\hline Irresponsabilidad & -.09 & .10 & .05 & .07 \\
\hline Manipulación & -.06 & .01 & .00 & .00 \\
\hline $\begin{array}{l}\text { Desregulación } \\
\text { Perceptual }\end{array}$ & $-.13^{* *}$ & .01 & .0 & .05 \\
\hline Perseveración & $-.13^{* *}$ & .06 & .03 & .04 \\
\hline Afectividad restringida & $-.13^{* *}$ & .08 & .00 & .07 \\
\hline Perfeccionismo & -.09 & .00 & .01 & -.02 \\
\hline Toma de riesgos & -.04 & -.03 & .02 & -.03 \\
\hline $\begin{array}{l}\text { Inseguridad de } \\
\text { Separación }\end{array}$ & -.06 & -.04 & .03 & .00 \\
\hline Sumisión & -.01 & .03 & -.04 & .04 \\
\hline Recelo & $-.15^{\star *}$ & .09 & .07 & $.14^{* *}$ \\
\hline $\begin{array}{l}\text { Creencias y } \\
\text { Experiencias Inusuales }\end{array}$ & $-.16^{\star *}$ & .02 & .07 & .05 \\
\hline Aislamiento & $-.11^{* *}$ & .10 & .07 & $.15^{* \star}$ \\
\hline
\end{tabular}

\section{DISCUSIÓN}

El objetivo de este trabajo fue analizar los rasgos disfuncionales de personalidad propuestos en la sección III del DSM-5 (American Psychiatric Association, 2013a) en relación con el sexo, tipo de carrera y rendimiento académico en una muestra de estudiantes universitarios de Buenos Aires. En función de los resultados, se verificaron las hipótesis que postulaban diferencias según sexo, tipo de carrera, así como su vínculo con el rendimiento académico. 
Comenzando por las diferencias según sexo, las mujeres presentaron medias superiores en los puntajes del dominio Afecto Negativo, así como en sus correspondientes rasgos de Ansiedad y Labilidad Emocional. También exhibieron medias mayores en Impulsividad. Los hombres, por su parte, evidenciaron mayores puntajes en el dominio de Antagonismo (y sus rasgos de Búsqueda de Atención, Insensibilidad, Engaño, Grandiosidad, Manipulación). También en Toma de Riesgo (perteneciente al dominio Desinhibición) y Excentricidad (correspondiente al dominio de Psicoticismo). Estos resultados son coincidentes con investigaciones previas efectuadas desde la clasificación vigente de los trastornos de personalidad de la sección II, ya que, tal como informaron Micin y Bagladi (2011), los hombres presentaron trastornos de personalidad más frecuentemente que las mujeres, del mismo modo que en este trabajo los estudiantes de sexo masculino exhibieron puntajes superiores a las mujeres en variadas escalas. Si bien esto no permite diagnosticar un trastorno de personalidad como en los estudios previos, cabe destacar que presentan medias mayores en el criterio B (American Psychiatric Association, 2013a). También resultan similares a los hallazgos de Bastiaens et al. (2015) quienes encontraron puntuaciones superiores en Afecto Negativo en las mujeres y mayores en Antagonismo en los hombres, corroborándose que las primeras se caracterizan por la rápida expresión de emociones negativas, mientras que los estudiantes de sexo masculino realizan comportamientos guiados por menor empatía y con el objetivo de obtener beneficios para sí mismos por sobre los demás. Respecto del vínculo con el modelo de los Cinco Factores, como ya se mencionó, investigaciones previas informaron que las mujeres reportan mayor neuroticismo (e.g., Chapman et al., 2007; Kajoniusa \& Johnson, 2018; Rantanen et al., 2007), el cual está ligado teóricamente al Afecto Negativo, dominio con puntaje elevado de dicho grupo.

Al considerar las diferencias según tipo de carrera, variable poco explorada en la literatura previa, se detectó un patrón de diferencias en los cuáles los estudiantes de Ciencias de la Salud reportaron puntuaciones menores en los rasgos disfuncionales al ser comparados con estudiantes de otras carreras y, dentro de estas, los educandos pertenecientes al área de Ciencias Básicas-Aplicadas exhibieron medias superiores. Esto es coincidente con el estudio de Choteau et al. (2016), en el que los alumnos de Ciencias presentaron rasgos autistas y esquizoides en comparación con los estudiantes de Humanidades.

Por último, con relación al vínculo con los diversos índices de rendimiento académico, también se observaron dos patrones de asociaciones. Por un lado, las asociaciones negativas con el índice de aprobación dan cuenta que, a mayor presencia de rasgos disfuncionales, menor rendimiento. Por otro lado, los indices de aplazos, asignaturas libres y recursadas, exhibieron asociaciones positivas, implicando que peores desempeños se presentan juntamente con caracteristicas disfuncionales de personalidad. Los datos siguen la misma dirección que los informados en trabajos previos que reportaron rendimiento inferior en alumnos que presentaban diversos trastornos de personalidad (e.g., Bagge et al., 2004; Hunt et al., 2010; Krajniak et al., 2017) y sintomatologia psicopatológica (Urquijo et al., 2013; Valencia-Molina et al., 2014). Cabe destacar que Desinhibición fue el dominio que presentó mayor cantidad de correlaciones estadísticamente significativas, lo cual resulta concordante con los numerosos estudios en torno al modelo de 
los Cinco Factores en los que se encontró que Responsabilidad -postulado como opuesto del dominio Desinhibición- constituía el factor de mayor importancia para predecir el rendimiento académico (e.g., Bergold \& Steinmayr, 2018; Dumfart \& Neubauer, 2016; Hakimi et al., 2011; Smrtnik Vitulic et al., 2011). A pesar de las similitudes con investigaciones previas, las correlaciones bajas dan cuenta de que los rasgos disfuncionales de personalidad no ocuparian un rol central entre las variables vinculadas al desempeño académico.

Como limitaciones de este estudio, debe mencionarse en primer lugar el muestreo intencional simple, por lo que los resultados son generalizables a sujetos con características sociodemográficas y académicas similares a las aquí descriptas. Si bien se eliminaron casos con protocolos con variables sociodemográficas incompletas, y respuestas al PID-5 con tendencias a responder en una sola dirección, al tratarse de una técnica que no dispone de escalas de validez -con las que se detecten tendencias a, por ejemplo, dar una imagen positiva o negativa de sí mismo- puede haber casos que presenten una distorsión en sus respuestas. Las medidas de rendimiento también fueron de autorreporte. Se espera que el amplio tamaño de la muestra haya logrado amortiguar posibles sesgos en algunos protocolos. Finalmente, debe mencionarse que la medición del rendimiento académico efectuada constituye una medida de varias posibles utilizadas en la temática (e.g., Garbanzo Vargas, 2014; Richardson et al., 2012; Rodríguez et al., 2004).

Como futuras líneas de investigación, sería conveniente analizar el modelo de rasgos disfuncionales en distintos ámbitos de aplicación, la cual constituye un área de vacancia. También sería de utilidad continuar explorando variables no intelectivas asociadas al desempeño académico, ya que los resultados encontrados en este estudio no son siempre concluyentes, como se ha detallado en la introducción teórica y considerando las correlaciones bajas aquí reportadas. En estudios recientes emergen otras variables no intelectivas plausibles de estudio, tales como la confianza confidence- que explica altos porcentajes de rendimiento (Stankov, 2013; Stankov, Morony \& Lee, 2014).

A pesar de las limitaciones, este trabajo realiza una primera aproximación al estudio del modelo dimensional de rasgos disfuncionales presentado en el DSM-5 (American Psychiatric Association, 2013a) en el ámbito educativo, analizando su vínculo con variables sociodemográficas y académicas, lo cual reviste importancia por la carencia de estudios a nivel internacional y local. La información aquí analizada permite delimitar el perfil de rasgos según sexo, así como según carrera, para analizar eventuales estrategias de intervención en las instituciones educativas.

\section{REFERENCIAS}

Agudelo Vélez, D. M., Casadiegos Garzón, C. P., \& Sánchez Ortíz, D. L. (2008). Características de ansiedad y depresión en estudiantes universitarios. International Journal of Psychological Research, 1(1), 34-39.

Al-Dajani, N., Gralnick, T. M., \& Bagby, R.M. (2015). A Psychometric Review of the Personality Inventory for DSM-5 (PID-5): Current Status and Future Directions. Journal of Personality Assessment, 98(1), 62-81. https://doi.org/10.1080/00223891.2015.1107572

American Psychiatric Association (2013a). Diagnostic and Statistical Manual of Mental Disorders (DSM-5). Washington D.C.: American Psychiatric Pub. https://doi.org/10.1176/appi.books.9780890425596

American Psychiatric Association (2013b). The Personality Inventory DSM-5 (PID-5) Self-Report Form (full version). Recuperado de https://www.psychiatry.org/psychiatrists/practice/dsm/educationalresources/assessment-measures\#Personality

Arrivillaga Quintero, M., Cortés García, C., Goicochea Jiménez, V. L., \& Lozano Ortiz, T. M. (2004). Caracterización de la depresión en jóvenes universitarios. Universitas Psychologica, 3(1), 17-25. 
Bach, B., Maples-Keller, J. L., Bo, S., \& Simonsen, E. (2016). The alternative DSM-5 personality disorder traits criterion: A comparative examination of three self-report forms in a danish population. Personality Disorders: Theory, Research, and Treatment, 7(2), 124-135. https://doi.org/10.1037/per0000162

Bagge, C., Nickell, A., Stepp, S., Durrett, C., Jackson, K., \& Trull, T. J. (2004). Borderline personality disorder features predict negative outcomes 2 years later. Journal of Abnormal Psychology, 113, 279288. https://doi.org/10.1037/0021-843X.113.2.279

Bastiaens, T., Claes, L., Smits, D., De Clercq, B., De Fruyt, F., Rossi, G., ... De Hert, M. (2016). The Construct Validity of the Dutch Personality Inventory for DSM-5 Personality Disorders (PID-5) in a Clinical Sample. Assessment, 23(1), 42-51. https://doi.org/10.1177/1073191115575069

Bergold, S., \& Steinmayr, R. (2018). Personality and intelligence interact in the prediction of academic achievement. Journal of Intelligence, 6(27), 1-18. https://doi.org/10.3390/jintelligence6020027

Campo Cabal, G., \& Gutiérrez Segura, J. C. (2001). Psicopatología en estudiantes universitarios de la Facultad de Salud - Univalle. Revista Colombiana de Psiquiatria, 30(4), 351-358.

Casullo, M. M., Morandi, P., \& Donati, F. (2006). Sintomas psicopatológicos, predisposición a perdonar y religiosidad en estudiantes universitarios. Anuario de Investigaciones, 13, 261-266.

Chabrol, H., Rousseau, A., Callahan, S., \& Hyler, S. (2007). Frequency and structure of DSM-IV personality disorder traits in college students. Personality and Individual Differences, 42(7), 1767-1776. https://doi.org/10.1016/j.paid.2007.05.015

Chapman, B. P., Duberstein, P. R., Sörensen, S., \& Lyness, J. M. (2007). Gender Differences in Five Factor Model Personality Traits in an Elderly Cohort: Extension of Robust and Surprising Findings to an Older Generation. Personality and Individual Differences, 43(6), 1594-1603. https://doi.org/10.1016/j.paid.2007.04.028

Choteau, L., Raynal, P., Goutaudier, N. \& Chabrol, H. (2016). Psychopathological traits in college students from top-ranking french schools: Do autistic features impair success in science when associated with schizotypal traits? Psychiatry Research, 237, 1-6. https://doi.org/10.1016/j.psychres.2016.01.038

Cohen, J. (1988). Statistical power analysis for the behavioral sciences. Second Edition. Hillsdale, NJ: LEA. https://doi.org/10.1002/bs.3830330104

Costa, P. T., \& McCrae, R. R. (1985). The NEO Personality Inventory Manual. Odessa Florida: Psychological Assessment Resources. https://doi.org/10.1037/t07564-000

De Fruyt, F., De Clercq, B., De Bolle, M., Wille, B., Markon, K., \& Krueger, R. F. (2013). General and maladaptive traits in a five-factor framework for DSM-5 in a university student sample. Assessment, 2O(3), 295-307. https://doi.org/10.1177/1073191113475808

DeYoung, C. G., Grazioplene, R. G., \& Peterson, J. B. (2012). From madness to genius: The Openness/Intellect trait domain as a paradoxical simplex. Journal of Research in Personality, 46, 6378. https://doi.org/10.1016/j.jrp.2011.12.003

Dumfart, B., \& Neubauer, A. C. (2016). Conscientiousness Is the Most Powerful Noncognitive Predictor of School Achievement in Adolescents. Journal of Individual Differences, 37(1), 8-15. https://doi.org/10.1027/1614-0001/a000182

Eisenberg, D., Gollust, S. E., Golberstein, E., \& Hefner, J. L. (2007). Prevalence and correlates of depression, anxiety and suicidality among university students. American Journal of Orthopsychiatry, 77(4), 534-542. https://doi.org/10.1037/0002-9432.77.4.534

Ekselius, L., Tillfors, M., Furmark, T., \& Frederikson, M. (2001). Personality disorders in the general population: DSM-IV and ICD-10 defined prevalence as related to sociodemographic profile. Personality and Individual Differences, 30, 311-320. https://doi.org/10.1016/S0191-8869(00)00048-9

Finch, H., \& French, B. (2013). A Monte Carlo comparison of robust manova test statistics. Journal of Modern Applied Statistical Methods, 12(2), 35-81. https://doi.org/10.22237/jmasm/1383278580

Garbanzo Vargas, G. M. (2007). Factores asociados al rendimiento en estudiantes universitarios, una reflexión desde la calidad de la educación superior pública. Revista Educación, 31(1), 43-63. https://doi.org/10.15517/revedu.v31i1.1252

Góngora, V. C., \& Castro Solano, A. (2017). Pathological Personality Traits (DSM-5), Risk Factors, and Mental Health. SAGE Open, 1-10. https://doi.org/10.1177/2158244017725129

Gore, W. L., \& Widiger, T. A. (2013). The DSM-5 dimensional trait model and five-factor models of general personality. Journal of Abnormal Psychology, 122(3), 816-21. https://doi.org/10.1037/a0032822

Gutiérrez, F., Aluja, A., Peri, J. M., Calvo, N., Ferrer,M., Baillés, E., Gutiérrez-Zotes, J. A., Gárriz, M., Caseras, X., Markon, K. E., \& Krueger, R. F. (2015). Psychometric Properties of the Spanish PID-5 in a Clinical and a Community Sample. Assessment, 24(3), 326-336. https://doi.org/10.1177/1073191115606518

Hair, J. F., Anderson, R. E., Tatham, R. L. \& Black, W. C. (1999). Análisis multivariante. Madrid: Prentice Hall.

Hakimi, S., Hejazi, E., \& Lavasani, M. G. (2011). The relationships between personality traits and students' academic achievement. Social and Behavioral Sciences, 29, 836-845. https://doi.org/10.1016/j.sbspro.2011.11.312

Heath, L. M., Drvaric, L., Hendershot, C. S., Quilty, L. C., \& Bagby, R. M. (2018). Normative and Maladaptive Personality Trait Models of Mood, Psychotic, and Substance Use Disorders. Journal of Psychopathology and Behavioral Assessment, 40(4), 606-613. https://doi.org/10.1007/s10862-0189688-0

Hunt, J., Eisenberg, D., \& Kilbourne, A. (2010). Consequences of receipt of a psychiatric diagnosis for completion of college. Psychiatric Services, 61, 399-404. https://doi.org/10.1176/ps.2010.61.4.399

Kajoniusa, P. J., \& Johnson, J. (2018). Sex differences in 30 facets of the five factor model of personality in the large public $(\mathrm{N}=320,128)$. Personality and Individual Differences, 129, 126-130. 
https://doi.org/10.1016/j.paid.2018.03.026

Krajniak, M. I., Pievsky, M., Eisen, A. R., \& McGrath, R. E. (2017). The relationship between personality disorder traits, emotional intelligence, and college adjustment. Journal of Clinical Psychology, 1-14. https://doi.org/10.1002/jclp.22572

Krueger, R. F. (2013). Personality disorders are the vanguard of the Post-DSM-5.0 era, Personality Disorders: Theory, Research, and Treatment, 4(4), 355-362. https://doi.org/10.1037/per0000028

Krueger, R. F., Derringer, J., Markon, K. E., Watson, D., \& Skodol, A. E. (2012). Initial construction of a maladaptive personality trait model and inventory for DSM-5. Psychological Medicine, 42, 1879-1890. https://doi.org/10.1017/S0033291711002674

Micin, S., \& Bagladi, V. (2011). Salud mental en estudiantes universitarios: incidencia de psicopatologia y antecedentes de conducta suicida en población que acude a un servicio de salud estudiantil. Terapia Psicológica, 29(1), 53-64. https://doi.org/10.4067/S0718-48082011000100006

Ministerio de Educación (2013). Anuario 2013. Estadística universitarias argentinas. Buenos Aires: Subsecretaria de Gestión y Coordinario de Políticas Universitarias.

Pardo, A., \& San Martín, R. (1999). Análisis de datos en psicología II. Madrid: Pirámide.

Poropat, A. E. (2009). A meta-analysis of the Five-Factor Model of personality and academic performance. Psychological Bulletin, 135(2), 322-338. https://doi.org/10.1037/a0014996

Rantanen, J., Metsäpelto, R., Feldt, T., Pulkkinen, L., \& Kokko, K. (2007). Long-term stability in the Big Five personality traits in adulthood. Scandinavian Journal of Psychology, 48, 511-518. https://doi.org/10.1111/j.1467-9450.2007.00609.x

Rial Boubeta, A. R., Varela Mallou, J., \& Tejada, A. J. R. (2001). Depuración y análisis preliminares de datos en SPSS. Madrid: Ra-ma.

Richardson, M., Abraham, C., \& Bond, R. (2012). Psychological correlates of university students' academic performance: A systematic review and meta-analysis. Psychological Bulletin, 138(2), 353-387. https://doi.org/10.1037/a0026838

Rodríguez, S., Fita, E., \& Torrado, M. (2004). El rendimiento académico en la transición secundaria universidad. Revista de Educación, 334, 391-414

Smrtnik Vitulic, H., \& Prosen, S. (2011). Personality and cognitive abilities as predictors of university students' academic achievement. Journal for General Social Issues, 21(3), 715-732. https://doi.org/10.5559/di.21.3.06

SPSS Inc. (2009). PASW Statistics for Windows, Version 18.0. Chicago: SPSS Inc.

Stankov, L. (2013). Noncognitive predictors of intelligence and academic achievement: An important role of $\begin{array}{llll}\text { confidence. Personality and Individual Differences, 55(7), 727-732. } & \text {. }\end{array}$ https://doi.org/10.1016/j.paid.2013.07.006

Stankov, L., Morony, S., \& Lee, Y. P. (2014) Confidence: the bestnon-cognitive predictor of academic achievement? Educational Psychology: An International Journal of Experimental Educational Psychology, 34(1), 9-28. https://doi.org/10.1080/01443410.2013.814194

Stover, J. B., \& Bruno, F. E. (2016). Ajuste psicológico, motivación y rendimiento académico en el ámbito universitario. En VIII Congreso Internacional de Investigación y Práctica Profesional en Psicología XXIII Jornadas de Investigación de la Facultad de Psicologia XII Encuentro de Investigadores (p. 45). Buenos Aires, Argentina: Facultad de Psicologia, Universidad de Buenos Aires.

Stover, J. B., Castro Solano, A., \& Fernández Liporace, M. (2019). Dysfunctional personality traits: Its relationship with Five Factor Model, adaptation and symptomatology in a community sample from Buenos Aires. Research in Psychotherapy: Psychopathology, Process and Outcome, 22(2), 281-291. https://doi.org/10.4081/ripppo.2019.343

Thomas, K. M., Yalch, M. M. Krueger, R. F., Wright, A. G. C., Markon, K. E., \& C. J., Hopwood (2013). The convergent structure of DSM-5 personality trait facets and Five-Factor Model trait domains. Assessment, 2O(3), 308-311. https://doi.org/10.1177/1073191112457589

Urquijo, S., del Valle, M., Ané, A., Heredia, L., \& Martinez, B. (Agosto, 2013). Psicopatología y desempeño académico en estudiantes universitarios. Trabajo presentado en la XIV Reunión Nacional y III Encuentro Internacional de la Asociación Argentina de Ciencias del Comportamiento. Asociación Argentina de Ciencias del Comportamiento AACC, Córdoba.

Valencia-Molina, A. M., Pareja-Galvis, A. M., \& Montoya Arenas, D. A. (2014). Trastornos neuropsiquiátricos en estudiantes universitarios con bajo rendimiento académico de una universidad privada de Medellín. CES Psicología, 7(1), 69-78.

Widiger, T. A. (2011). Integrating normal and abnormal personality structure: A proposal for DSM-V. Journal of Personality Disorders, 25(3), 338-363. https://doi.org/10.1521/pedi.2011.25.3.338

\section{Recibido 01-02-2019 | Aceptado 18-09-2019}

Este trabajo se encuentra bajo una Licencia Creative Commons Atribución 4.0 Internacional que permite a terceros utilizar lo publicado siempre que se dé el crédito pertinente a los autores y a Psicodebate 